\title{
Group mutual exclusion in linear time and space
}

\author{
Yuan $\mathrm{He}^{\mathrm{a}}$, K. Gopalakrishnan ${ }^{\mathrm{b}, *}$, Eli Gafni ${ }^{\mathrm{a}}$ \\ a Department of Computer Science, University of California at Los Angeles, Los Angeles, CA-90095, United States \\ ${ }^{\mathrm{b}}$ Department of Computer Science, East Carolina University, Greenville, NC-27858, United States
}

\section{A R T I C L E I N F O}

\section{Article history:}

Received 1 May 2016

Received in revised form 12 April 2017

Accepted 30 May 2017

Available online 7 June 2017

\section{Keywords:}

Mutual exclusion

Group mutual exclusion

Remote memory reference complexity

Lamport's Bakery Algorithm

Black and White Bakery Algorithm

\begin{abstract}
A B S T R A C T
We present two algorithms for the Group Mutual Exclusion (GME) Problem that satisfy the properties of Mutual Exclusion, Starvation Freedom, Bounded Exit, Concurrent Entry and First Come First Served. Both our algorithms use only simple read and write instructions, have $O(N)$ Shared Space complexity and $O(N)$ Remote Memory Reference (RMR) complexity in the Cache Coherency (CC) model. Our first algorithm is developed by generalizing the well-known Lamport's Bakery Algorithm for the classical mutual exclusion problem, while preserving its simplicity and elegance. However, it uses unbounded shared registers. Our second algorithm uses only bounded registers and is developed by generalizing Taubenfeld's Black and White Bakery Algorithm to solve the classical mutual exclusion problem using only bounded shared registers. We show that contrary to common perception our algorithms are the first to achieve these properties with this combination of complexities.
\end{abstract}

(C) 2017 The Authors. Published by Elsevier B.V. This is an open access article under the CC BY-NC-ND license (http://creativecommons.org/licenses/by-nc-nd/4.0/).

\section{Introduction}

Mutual Exclusion is a classical problem in distributed computing introduced by Dijkstra in 1965 [4]. The Group Mutual Exclusion (GME) problem, introduced by Joung in 2000 [10], is a natural generalization of the classical mutual exclusion problem.

Before formally stating the problem, we first motivate the generalization by the following illustrative example. In university environment we can think of the critical section as a room allocated by the University to students of all faiths to pray in the most neutral policy, FCFS (First-Come First-Serve). Naturally (to believers!), students of different faith cannot use the room to pray concurrently. However, students of the same faith are encouraged to pray concurrently. Since students pray individually, a student cannot overtake another waiting student to enter the prayer room just because a member of her faith is currently praying. This will prolong the waiting of the waiting student. Yet, students who arrive while only others of the same denomination are present can enter and pray without waiting. We assume that open minded Christian, or secular who might call to God at a dire strait, may pick a different denomination from time to time, though, they have to declare their pick upon arrival. If a member of different declared denomination is present, then service is FCFS. Students negotiate entrance to the prayer room by writing on a blackboard, intermittently taking a break from waiting to have coffee, and then come back to check the negotiated queue. Henceforth a student is a process, and each student depending on UID, has a space allocated on the blackboard to be written in exclusion.

\footnotetext{
* Corresponding author.

E-mail addresses: heyuan89@cs.ucla.edu (Y. He), gopal@ecu.edu (K. Gopalakrishnan), eli@ucla.edu (E. Gafni).
} 
In Group Mutual Exclusion problem, processes repeatedly cycle through four sections of code viz., Remainder Section, Entry Section, Critical Section (CS) and Exit Section, in that order. An execution of the last three sections will be called an invocation. A process picks a session number when it leaves the remainder section and this session number can be different in each invocation. A process is said to be an active process, if it is in one of its invocations. Two active processes are in conflict if their session numbers are different. Unlike the classical mutual exclusion problem, multiple processes are allowed to be in the critical section at the same time, provided they are not conflicting. In fact, in the presence of active processes all of which are mutually non-conflicting, entry into the critical section takes bounded number of process steps. Formally, the problem consists of designing code for the entry section and the exit section such that the following four properties are satisfied.

P1 Mutual exclusion: No two conflicting processes can be in the critical section at the same time.

P2 Starvation freedom: If no process stays in the critical section forever, then any process that enters the entry section eventually enters the critical section.

P3 Bounded exit: After entering the exit section, a process is guaranteed to leave it within a bounded number of its own steps.

P4 Concurrent entry: In the absence of conflicting processes, a process in the entry section should be guaranteed to enter the critical section within a bounded number of its own steps.

The Concurrent Entry property is crucial to the GME problem. It was stated informally by Joung [10] and then later formalized by Hadzilacos [6]. The intent of this property is to ensure concurrency: active processes that request the same session, in the absence of conflicting processes, should be allowed to enter the CS without unnecessary synchronization among themselves.

In this paper, we will require that the algorithm satisfies the First Come First Served (FCFS) property in addition to the above four properties. The standard way to formalize this is to split the entry section into two sections viz., Doorway Section and Waiting Room Section. The doorway section of the code is free of "wait" statements, i.e., it can be completed by a process in a bounded number of its own steps. The waiting room section is where the actual synchronization with other conflicting processes occurs and may entail indefinite waiting. The notion of doorway was originally introduced by Lamport [12] in the context of classical mutual exclusion problem. We would say that process $P_{i}$ doorway precedes process $P_{j}$, if $P_{i}$ completes the doorway before $P_{j}$ enters the doorway. Now, the FCFS property can be formally stated as given below.

P5 FCFS: If process $P_{i}$ doorway precedes process $P_{j}$ and the two processes request different sessions, then process $P_{j}$ does not enter the critical section before process $P_{i}$.

If neither $P_{i}$ doorway precedes $P_{j}$ nor $P_{j}$ doorway precedes $P_{i}$, we would say the two processes are doorway concurrent.

\subsection{Model}

We consider a system consisting of $N$ processes, named $P_{1}, P_{2} \ldots P_{N}$ and a set of shared variables. Each process also has its own private variables. Processes can communicate only by writing into and reading from shared variables. An execution is modeled as a sequence of process steps. In each step, a process performs some local computation or reads from a shared variable or writes into a shared variable. We assume that these steps of reading or writing are atomic. The processes take steps asynchronously. Specifically, this means that an unbounded number of steps of some other process could be performed in between two consecutive steps of a process. We assume that our processes are live; this means that if a process is active it will eventually execute its next step.

We allow only simple read and write operations on shared variables. We assume that these read and write operations are atomic. However, we do not assume that processes have access to more powerful synchronization operations such as atomic test-and-set and compare-and-swap.

There are two general architectural paradigms considered for shared memory in the literature, viz., Distributed Shared Memory (DSM) Model and Cache-Coherency (CC) Model. In the DSM model, each processor has its own memory module and each shared variable is assigned to a particular processor. When a processor is referencing a shared variable, it is locally accessible, if it is allocated to that processor itself; on the other hand, it is a remote access, if it is allocated to some other processor. In the CC model, all shared variables are stored in a global memory module that is not associated with any particular processor. Each processor also has a local cache and some hardware protocol ensures cache coherence, i.e., all copies of the same variable in different local caches are consistent. Every time a process reads a shared variable, it does so using a local (cached) copy of the variable. A local copy of the variable may not be valid, if the process has never read the variable before or if some process overwrote it in the global memory module. Whenever, upon reading a cached variable, the process is informed by the system that its value is invalid, it makes a remote memory reference and migrates the variable to its local cache. We assume that once a shared variable is brought into a process's local cache it remains there indefinitely. Also, every time a process writes a shared variable, the process writes the variable in the global memory module, which involves a remote memory reference. Note that, this action invalidates all cached copies of that variable. In 
this paper, we will be working exclusively under the cache-coherency model, a model of practical significance considering that virtually every modern multi-processor is cache-coherent.

By the term time complexity, we mean Remote Memory Reference (RMR) complexity in this paper. It is simply the worst case number of remote memory references performed by a process in one invocation. This is because remote memory references are the most time consuming operations as they involve interconnect traversal. Also, counting the number of remote memory references is probably the only reasonable way to evaluate the time complexity of mutual exclusion algorithms, since plain steps complexity of mutual exclusion algorithms is unbounded. An algorithm is called a local-spin algorithm if the maximum number of remote memory references made in any invocation is bounded.

By the term space complexity, we mean the total amount of shared space a solution entails. We do not count the private variables when measuring space complexity. There are two different situations of interest, one in which the shared variables are unbounded registers and the other in which the shared variables are bounded registers.

\subsection{Our contribution and related work}

Joung's original algorithm for the GME problem satisfies the four basic properties. It does not satisfy the FCFS property. Moreover, it has unbounded RMR complexity. Hadzilacos (see [6]) gave the first solution for the GME problem that has the FCFS property. His algorithm can be thought of as a modular composition of two independent algorithms, one, "the FCFS algorithm" provides FCFS property (but not necessarily guarantees mutual exclusion) and the other, "the ME algorithm" provides mutual exclusion property (but not necessarily FCFS). This algorithm has shared space complexity of $\Theta\left(N^{2}\right)$. It was (mistakenly in hindsight, see Section 4) claimed that the algorithm has RMR complexity of $O(N)$ in the CC model. The algorithm uses only bounded shared variables and simple read and write operations. It was left as an open problem to develop a solution (satisfying P1 through P5) for the GME problem that runs in linear time and space using only bounded shared variables.

Subsequently, in [8], Jayanti et al. presented an algorithm presumed to be of linear time and space, solving the challenge of Hadzilacos. Jayanti et al. retained the idea of modular composition and also the "ME algorithm" that Hadzilacos used. They came up with a clever modification to the "FCFS algorithm" of Hadzilacos to reduce the space complexity to $\Theta(N)$. Although they did not explicitly claim so, their algorithm was also deemed to have the linear RMR Complexity in view of the wrong claim by Hadzilacos. ${ }^{1}$

Both works use a slightly modified version of a classical mutual exclusion algorithm developed independently by Burns [2] and Lamport [13] as the "ME algorithm". This is an elegant algorithm that uses just one bit of shared space per process. In Section 4, we show that this algorithm actually has an intricate structure and has the worst case RMR complexity of $\Omega\left(N^{2}\right)$. It follows that algorithms of both Hadzilacos and Jayanti et al. are of RMR complexity $\Omega\left(N^{2}\right)$. Hence, the challenge posed by Hadzilacos has yet to be met. Our observation, and part of our contribution, that the challenge is still on, initiated this paper.

Our first algorithm, presented in Section 2, is a generalization of the classic Lamport's Bakery Algorithm to solve the GME problem while maintaining its simplicity and elegance. It uses unbounded registers to solve the GME problem (satisfying P1 through P5) and runs in linear time and space.

Takamura and Igarashi also made an attempt in [14] to generalize Lamport's Bakery Algorithm to solve the GME problem. They presented three different algorithms in that paper. However, none of their algorithms satisfies the concurrent entry property nor the FCFS property.

In 2004 (which is after the publication of [6] and [8]), Taubenfeld [15] came up with an elegant algorithm called Black and White Bakery Algorithm that solves the classical mutual exclusion problem with only bounded shared registers. His approach is a lot simpler than a prior method that bounds the registers of the Lamport's Bakery Algorithm developed by Jayanti et al. in [9]. Our second algorithm, presented in Section 6 is a generalization of the Black and White Bakery Algorithm to solve the GME problem. Our algorithm satisfies the properties P1 through P5 and runs in linear time and space using bounded shared registers. Thus, our algorithm is the first one to solve the open problem originally posed by Hadzilacos, a decade and a half ago.

We present a comparison of different GME algorithms in Table 1. To make the comparison fair, we include only those algorithms that solve the problem using only simple read/write instructions. The first row in the table describes the properties of Joung's original algorithm for the GME problem. The next four rows compare the algorithms for the GME problem that use unbounded shared registers. The last four rows compare the algorithms for the GME problem that use bounded shared registers.

\section{Generalizing Lamport's Bakery Algorithm}

In this section, we present a very simple algorithm for the GME problem by generalizing Lamport's Bakery Algorithm (see [12]) for the classical mutual exclusion problem. This algorithm is based on the method commonly used in bakeries, in

\footnotetext{
1 For example, the recent paper by Bhatt and Huang [1] explicitly states that the RMR complexity of the algorithm by Jayanti et al. is $O(N)$.
} 
Table 1

Comparison of GME algorithms that use only simple read/write instructions.

\begin{tabular}{|c|c|c|c|c|c|c|c|}
\hline Algorithm & RMR & Space & P2? & P3? & P4? & P5? & Bounded? \\
\hline Joung [10] & $\infty$ & $\mathrm{O}(\mathrm{N})$ & Yes & Yes & Yes & No & Yes \\
\hline Takamura et al. [14] I & $\infty$ & $\mathrm{O}(\mathrm{N})$ & No & Yes & No & No & No \\
\hline Takamura et al. [14] II & $\mathrm{O}(\mathrm{N})$ & $\mathrm{O}(\mathrm{N})$ & Yes & No & No & No & No \\
\hline Takamura et al. [14] III & $\mathrm{O}(\mathrm{N})$ & $\mathrm{O}(\mathrm{N})$ & Yes & No & No & No & No \\
\hline This work (GLB Algorithm) & $\mathrm{O}(\mathrm{N})$ & $\mathrm{O}(\mathrm{N})$ & Yes & Yes & Yes & Yes & No \\
\hline Keane \& Moir [11] & $\mathrm{O}(\log N)$ & $\mathrm{O}(\mathrm{N})$ & Yes & No & No & No & Yes \\
\hline Hadzilacos [6] & $\mathrm{O}\left(N^{2}\right)$ & $\mathrm{O}\left(N^{2}\right)$ & Yes & Yes & Yes & Yes & Yes \\
\hline Jayanti et al. [8] & $\mathrm{O}\left(N^{2}\right)$ & $\mathrm{O}(\mathrm{N})$ & Yes & Yes & Yes & Yes & Yes \\
\hline This work (BWBGME Algorithm) & $\mathrm{O}(\mathrm{N})$ & $\mathrm{O}(\mathrm{N})$ & Yes & Yes & Yes & Yes & Yes \\
\hline
\end{tabular}

which a customer receives a token number upon entering the store and the holder of the lowest token number is the next one served. We will refer to it as Generalized Lamport's Bakery (GLB) Algorithm.

The algorithm is presented in Fig. 1. It uses three shared variables. The first one Session is an integer array of size $N$ and Session $[i]$ indicates the session number that process $P_{i}$ requests in the current invocation to enter the CS. The second one is Token, an integer array of size $N$ and Token[i] represents the token number selected by process $P_{i}$. The third shared variable is Choosing, a boolean array of size $N$ and Choosing $[i]$ is true would indicate that process $P_{i}$ is currently attempting to make a new request to enter the critical section. The Session array and the Token array are initialized to zero and the Choosing array is initialized to false. The doorway of the algorithm consists of lines 3-6 and the waiting room is made up of lines 7-10.

When a process leaves the remainder section, it first sets the variable Choosing $[i]$ to true to signal other processes that it is about to make a new request to enter the critical section. Next, it places the desired session number in Session[i]. We assume that all session numbers are positive integers. It then selects its token number to be one more than the maximum of the token numbers of all other processes and places it in Token[i]. Finally, $P_{i}$ sets Choosing[i] to false to signal other processes that it has completed the doorway section for the new request.

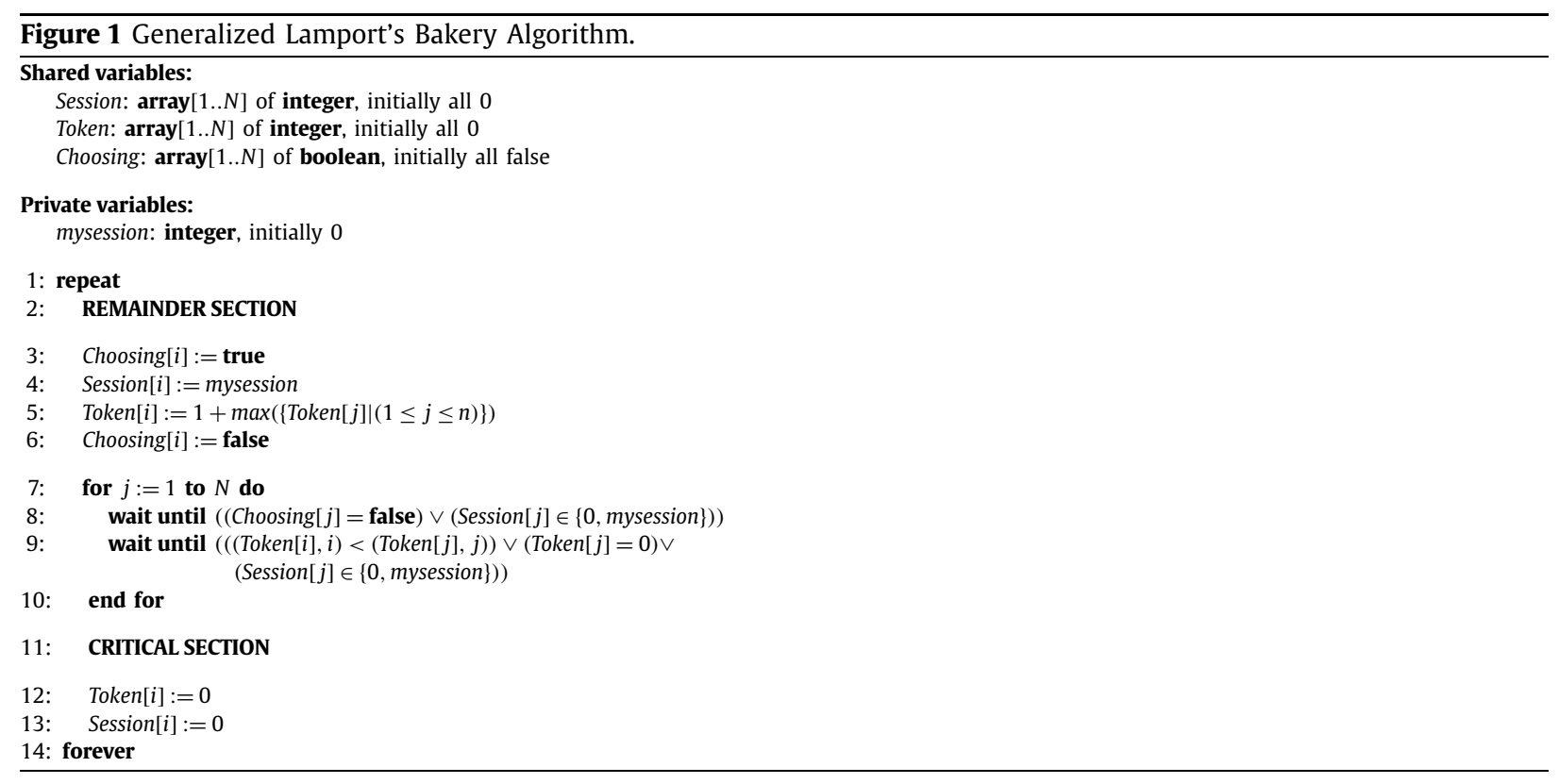

In the waiting room, at line 8 , for each other process $P_{j}$, process $P_{i}$ checks to see whether Session[j] is zero or same as mysession. In either case, there is no problem and so $P_{i}$ can move on. On the other hand, if process $P_{j}$ is requesting a conflicting session (i.e., Session $[j] \notin\{0$, mysession $\}$ ), $P_{i}$ waits for $P_{j}$ to finish the doorway and set Choosing $[j]$ to be false. Next, at line 9 , process $P_{i}$ waits on each conflicting process $P_{j}$ (i.e., Session[j] $\notin\{0$, mysession $\}$ ) until either $P_{j}$ has exited the critical section (i.e., $\operatorname{Token}[j]=0)$ or $P_{j}$ has a larger token number (i.e., ((Token $\left.[i], i\right)<(\operatorname{Token}[j], j)$ )). It is possible that two conflicting processes pick the same token number. In that case, we use the process identifier to resolve the ties (line 9). The relation "less than" among ordered pairs of integers is defined as in the Lamport's Bakery Algorithm. That is $(a, b)<(c, d)$ if $a<c$ or if $a=c$ and $b<d$ (a, b, c and d are all integers). For simplicity, if Token[i] =Token[j], we say the process with 
the smaller process identifier has the smaller token number. After the loop, process $P_{i}$ enters the CS. In the exit section, $P_{i}$ resets Token[i] to 0 and then Session[i] to 0 .

\section{Proof of correctness of the generalized Lamport's Bakery Algorithm}

The algorithm satisfies the mutual exclusion property, starvation freedom property, bounded exit property, concurrent entry property and the FCFS property. Here we present a complete proof that the algorithm satisfies these properties.

\section{Lemma 1. The GLB algorithm has the FCFS property.}

Proof. Assume two conflicting processes $i$ and $j$ request the CS. Also assume that process $i$ finishes the doorway before process $j$ starts the doorway. So, when process $j$ is computing its token number, it will read the token number of process $i$ and select a larger token number. As process $j$ has a larger token number, when process $j$ checks with process $i$ at line 9 , it will wait for process $i$ to exit the CS.

\section{Lemma 2. The GLB algorithm has the Mutual Exclusion property.}

Proof. Suppose two conflicting processes $i$ and $j$ are in the CS at the same time with different sessions. By Theorem 1 , process $i$ and process $j$ must be doorway-concurrent. Without loss of generality, we assume process $i$ enters the CS first. Since processes $i$ and $j$ are doorway concurrent, process $j$ has at least finished executing line 3 when process $i$ checks on process $j$ at line 8 .

If process $j$ has also finished updating Session[ $j$ ] by that time, process $i$ will wait for process $j$ to finish the doorway, as they are requesting conflicting sessions. Since process $i$ enters the CS first, it must have a smaller token number than process $j$. As a consequence, at line 9, process $j$ will wait for process $i$ because it finds that process $i$ has a smaller token number. Therefore, process $j$ cannot enter the CS until process $i$ finishes the CS and resets its token number, which is contradicting with our assumption.

On the other hand, if process $j$ has not finished updating Session[ $j$ ] by that time, then process $i$ will not wait on process $j$ in line 8 as Session $[j]=0$. However, if this is the case, process $i$ has already selected its token number whereas process $j$ has not yet begun selecting its token number. So, process $j$ 's token number is guaranteed to be larger than that of process $i$ and process $j$ will wait on process $i$ at line 9. Therefore, process $j$ cannot enter the CS until process $i$ finishes the CS and resets its token number, which is contradicting with our assumption.

Lemma 3. The GLB algorithm has the Bounded Exit property.

Proof. Since the exit section consists of two simple write instructions, a process that enters the exit section will trivially finish it within a bounded number of its own steps.

We now define another property called Deadlock Freedom. Deadlock freedom simply means deadlocks cannot occur in the system. Informally, Deadlock occurs in the system when one or more processes are "trying to enter" their critical sections, but no process ever does so. Lamport (see page 329 in [13]) has shown that under the assumptions that no process stays in the critical section forever (which we are assuming), there are only finitely many processes (which we are assuming) and the algorithm satisfies the "Bounded Exit property" (which we have just now shown is the case for our algorithm), the deadlock freedom property can be formally stated as follows:

P6 Deadlock Freedom If one or more processes are forever trying to enter their critical section with no success for any of them, then there exists a process that enters the critical section infinitely often.

Lemma 4. The GLB algorithm has the Deadlock Freedom property.

Proof. Suppose the algorithm does not satisfy the deadlock freedom property. Then there is an execution of the algorithm in which a nonempty set $S$ of processes enter the entry section but none of them enters the CS, and no process enters the CS infinitely often. We observe that no process can wait at line 8 forever since every process $j$ that requests the CS will finish the doorway eventually and set Choosing $[j]$ to false and process $j$ enters the CS only finitely many times. Therefore, processes in the set $S$ must wait on line 9 forever. There exists a process $i$ that has the smallest token number among all processes in $S$ and that process $i$ will pass all other processes at line 9 and enter the CS, which is a contradiction with our assumption.

It is easy to observe that the Starvation Freedom property immediately implies the Deadlock Freedom property. However, the converse is not necessarily true. Deadlock freedom means that the entire system of processes will always continue 
to make progress. However, it does not preclude the possibility of individual processes not making progress (i.e., waiting forever in the entry section).

Lemma 5. The GLB algorithm has the Starvation Freedom property.

Proof. Lamport has proved (see page 330 in [13]) that if an algorithm satisfies the deadlock freedom property and the FCFS property, then it necessarily satisfies the starvation freedom property. Hence, combining Lemma 1 and Lemma 4, we conclude that our algorithm has the starvation freedom property.

Lemma 6. The GLB algorithm has the Concurrent Entry property.

Proof. Assume that process $i$ requests a session, and no other process requests a different session. This means, for every other process $j$, it holds that Session $[j] \in\{0$, mysession $\}$. Since process $i$ always checks whether Session $[j] \in\{0, m y s e s s i o n\}$ in both the busy-wait loops in line 8 and line 9 , it cannot wait on either lines. So, it enters the CS within a bounded number of its own steps, thus satisfying the concurrent entry property.

We now analyze both the time and space complexity of the algorithm. The algorithm uses three shared variables, Session, Token and Choosing, each of which is an array of size $N$. So, obviously the algorithm has $\Theta(N)$ shared space complexity. However, the token numbers used by this algorithm will grow in an unbounded manner, just like in the Lamport's Bakery Algorithm.

We now analyze the time complexity of the algorithm. As said before, by time complexity, we mean the RMR complexity of the algorithm because remote memory references are the most time consuming operations. Recall that in the CC model, all shared variables are stored in a global memory module and processes migrate them to their local cache to access them. In the waiting room, there are only two loops viz., the busy-wait loops in line 8 and line 9 . In line 8 , when process $i$ is busy waiting for a process $j$, if Choosing $[j]$ changes to false, then process $i$ will immediately terminate the wait. It is possible that (before process $i$ observes the changed value of Choosing $[j]$ ) precess $j$ sets the Choosing $[j]$ to true again and requests another conflicting session. In that case, since process $i$ doorway-proceeds process $j$, process $j$ will get a larger token number than process $i$. So, process $j$ cannot enter the CS to finish that invocation and therefore cannot change the Session again until process $i$ finishes the CS. Thus, line 8 can only involve a maximum of five RMR (three for Choosing[ $j]$ and two for Session $[j])$. Similarly in line 9, when process $i$ is busy-waiting on Token[ $j]$, if Token[ $j$ ] changes, its new value will be either zero or a larger token number and in either case, process $i$ will terminate the wait. Also, any change in Session $[j]$ will also entail a change in Token $[j]$ and thus terminate the wait. Hence, line 9 involves a maximum of five RMR (one for Token[i], two for Token[ $j]$, two for Session $[j])$. There are only a constant number of RMR in line 8 and line 9 . As these two lines are enclosed within a for loop that can run a maximum of $N$ times, it follows that the entire waiting room is of $O(N)$ RMR complexity. Note that the doorway made up of line 3 through line 6 involves only a constant number of RMR, except for the implicit loop in line 5. The implicit loop in line 5 has $O(N)$ RMR complexity as it involves inspecting the token numbers of all other processes. Finally, it is easy to see that the exit section consisting of lines 12-13 involves exactly two RMR. Hence, the overall RMR complexity of this algorithm in the CC model is $O(N)$.

We now summarize the results in the form of the following Theorem.

Theorem 1. The Generalized Lamport's Bakery Algorithm presented in Fig. 1 solves the GME problem by satisfying all the five properties P1 through P5 in linear space (with unbounded registers) and time (RMR under the CC model) using only simple read and write operations.

An earlier paper by Takamura and Igarashi [14] also made an attempt to generalize Lamport's Bakery Algorithm to solve the GME problem. They presented three different algorithms in that paper. Their first algorithm is fairly simple, but does not satisfy the starvation freedom property. Their second and third algorithms do satisfy the starvation freedom property. However, apart from being quite complicated, they do not satisfy the bounded exit property. None of the three algorithms satisfies the FCFS property nor concurrent entry property. However, all three of their algorithms satisfy a weaker property known as concurrent occupancy in the literature (see [11] and [6]). The GLB algorithm shown in Fig. 1 is the simplest and most natural generalization of Lamport's Bakery Algorithm for the GME problem.

\section{A flaw in the literature}

We now look at attempts to solve the GME problem using only bounded shared registers and simple read and write operations. Two prominent algorithms in this regard are that of Hadzilacos [6] and that of Jayanti et al. [8]. Both of the algorithms can be viewed as a modular composition of an "FCFS Algorithm" and an "ME Algorithm". Not only that both of the algorithms use modular composition technique, but also they both use the same "ME Algorithm". Hadzilacos's algorithm is of space complexity $\Theta\left(N^{2}\right)$ and he claimed that his algorithm is of $O(N)$ RMR complexity in the CC model. Hadzilacos posed it as an open problem to devise an algorithm to solve the GME problem in linear time and space using only bounded 
Figure 2 Burns-Lamport ME algorithm.

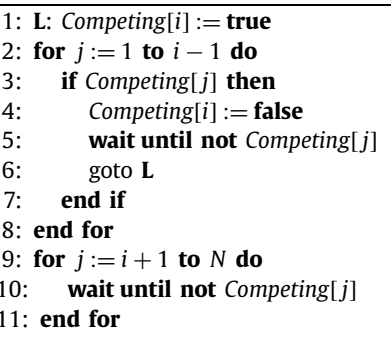

shared variables. Jayanti et al. came up with a clever modification to the "FCFS algorithm" of Hadzilacos and reduced the space complexity to $\Theta(N)$. We now show that the "ME Algorithm" they used is of complexity $\Omega\left(N^{2}\right)$ in the CC model thus establishing that neither of these two algorithms has $O(N)$ RMR complexity.

The "ME algorithm" used by them is independently discovered by Burns [2] and Lamport [13]. This algorithm is depicted in Fig. 2. In this algorithm Competing is a shared array of size $N$. Each element of the array is a boolean variable, initialized to false. The code given in Fig. 2 is for process $P_{i}$. The variable $j$ is a private variable.

We now briefly describe the algorithm. Every process $P_{i}$ owns a single bit Competing[i]. Only $P_{i}$ can write into Competing[i]; Other processes can read it. Before entering the CS, $P_{i}$ sets its bit to true and checks all lower numbered processes (lines 1-3). If any of them, say process $P_{j}$, is found to have set its bit to true, then process $P_{i}$ resets its bit to false, allowing the smaller-numbered process to make progress. It then waits for process $P_{j}$ 's bit to become false and then restarts the competition to enter the CS by going to line 1. Having checked all lower-numbered processes, process $P_{i}$ then checks the higher-numbered ones and waits for each of them to set its bit to false. However, this time while $P_{i}$ is waiting it does not set its bit to false. After that process $P_{i}$ enters the CS. It turns out that this simple algorithm guarantees mutual exclusion. We refer the reader to [13] (see also [2]) for a proof of correctness.

To analyze the RMR complexity under the CC Model, consider the following sequence of events:

1. Process $P_{N}$ sets its bit to true.

2. Process $P_{(N-1)}$ sets its bit to true.

3. Process $P_{N}$ checks all lower-numbered processes and finds that $P_{(N-1)}$ 's bit is set. So, $P_{N}$ sets its bit to false and waits for Competing $[N-1]$ to become false.

4. Process $P_{(N-2)}$ sets its bit to true.

5. Process $P_{(N-1)}$ checks all lower-numbered processes and finds that process $P_{(N-2)}$ 's bit is set. So, process $P(N-1)$ sets its bit to false and waits for Competing $[N-2]$ to become false.

6. Process $P_{N}$ now finds Competing[N-1] to be false and so restarts the competition by setting its bit to true.

7. Process $P_{N}$ checks all lower-numbered processes and finds that process $P_{(N-2)}$ 's bit is set. So, $P_{N}$ sets its bit to false and waits for Competing $[N-2]$ to become false.

8. Process $P_{(\mathrm{N}-3)}$ sets its bit to true.

9. Process $P_{(N-2)}$ checks all lower-numbered processes and finds that process $P_{(N-3)}$ 's bit is set. So, process $P(N-2)$ sets its bit to false and waits for Competing $[N-3]$ to become false.

10. Process $P_{N}$ now finds Competing $[N-2]$ to be false and so restarts the competition by setting its bit to true.

11. Process $P_{N}$ checks all lower-numbered processes and finds that process $P_{(N-3)}$ 's bit is set. So, $P_{N}$ sets its bit to false and waits for Competing $[N-3]$ to become false.

12. .

13. .

14.

15. Process $P_{N}$ checks all lower-numbered processes and finds that process $P_{1}$ 's bit is set. So, $P_{N}$ sets its bit to false and waits for Competing[1] to become false.

16. Process $P_{1}$ checks all higher-numbered processes and finds that all the bits are false and enters CS.

17. Process $P_{1}$ exits the CS and sets its bit to false.

Note that during the above sequence of events, process $P_{N}$ got blocked by each one of the lower numbered processes once. At the end of the above sequence, process $P_{1}$ 's request is satisfied. Also, at the end of the above sequence, the Competing bit of $P_{2}$ through $P_{N}$ are all false. Now, we can create a similar sequence of events, but this time with only processes $P_{2}$ through $P_{N}$ participating. We can recursively create a similar sequence of events again and again until finally we have only process $P_{N}$ participating.

The net effect is that in this worst case scenario, process $P_{N}$ got blocked by process $P_{(N-1)}$ a total of $(N-1)$ times, by process $P_{(N-2)}$ a total of $(N-2)$ times and so on. So, the total number of times that process $P_{N}$ gets blocked by some other processes is 


$$
\sum_{k=1}^{N-1} k=N(N-1) / 2=\Omega\left(N^{2}\right)
$$

In the CC model, at least one remote memory reference is involved each time a process gets blocked and hence the worst case RMR complexity of the algorithm in Fig. 2 is $\Omega\left(N^{2}\right)$ in the CC Model.

Therefore, the problem of developing a linear time (RMR) and linear (shared) space algorithm that uses only bounded shared variables for the GME problem, originally posed by Hadzilacos, is still open.

One might get the impression that we can immediately fix the problem, by plugging in some other mutual exclusion algorithm that has $O(N)$ RMR Complexity in place of Lamport-Burnst Mutual Exclusion Algorithm. Unfortunately, the situation is not that simple as we have to adapt the ME algorithm so that it provides concurrent entry for application in the development of GME algorithm using the modular composition technique. The Lamport-Burnst algorithm is easy to adapt by simply adding an extra condition to check whether the session number of the other process is the same as the session number of this process in all wait-until loops. On the other hand, the mutual exclusion algorithm of Taubenfeld [15] which has $O(N)$ RMR Complexity, as well as that of Yang and Anderson [16] which has $O(\log N)$ RMR Complexity, is not suitable for plugging in the modular design. Simply adding an extra condition to check the session number in all wait-until loops in these algorithms does not provide concurrent entry as they have more intricate structures. As far as we know, there is no mutual exclusion algorithm of $O(N)$ RMR complexity in the literature, that is easily adaptable to provide concurrent entry and can be plugged in the modular design. So, the problem of developing a linear time and linear space GME algorithm that uses only bounded registers is indeed a non-trivial problem. We develop such an algorithm in Section 6.

\section{Black and White Bakery Algorithm}

In 2004, Taubenfeld [15] came up with an elegant algorithm called Black and White Bakery Algorithm that solves the classical mutual exclusion problem with only bounded shared registers. In this section, we first review this algorithm and in the next section, we generalize the ideas developed in that paper and solve the GME problem with only bounded shared registers in linear time and space.

The key idea in this algorithm is to view the token as a colored token, i.e., the token has two components color and number. The color will be either black or white and the number will be a positive integer. As in Lamport's Bakery algorithm, we also use the Choosing shared variable. The algorithm also uses an additional shared bit variable called GlobalColor which can be either black or white. Unlike the other shared variables, GlobalColor is a multi-writer, multi-reader variable.

The algorithm is depicted in Fig. 3.

When process $P_{i}$ wants to enter the CS, it first sets the Choosing variable to true to notify other processes that it is attempting to pick its token. Then it reads the value of GlobalColor and sets its own token color to the read value. It then picks a number which is greater than the token numbers of all processes which have the same token color as that of $P_{i}$ After having selected the colored token, the process resets its Choosing variable to false to indicate to other processes that it is done with picking a token.

Once $P_{i}$ has got its colored token, it enters the waiting room and it waits until its colored token is the lowest and then enters the CS. The order between the colored tokens is defined as follows: If two tokens have the same color, the token with the smaller number is smaller. If two token have different colors, the token whose color is different from the GlobalColor is smaller. If two processes have the same token color and the same token number, the process identifiers are used to break the tie as in Lamport's Bakery algorithm.

When $P_{i}$ is through with the CS, it sets the shared variable GlobalColor to the opposite of its own token color and then resets its own token number to zero. The setting of GlobalColor to the opposite color is to ensure that priority is given to waiting processes whose token color is the same as the one that $P_{i}$ held.

If at a certain point of time $t$, the GlobalColor has a value of $c$, then the algorithm ensures that all processes with token color different from $c$ that are in the entry section at time $t$ enter the CS before any process with a token color of $c$.

After each time the GlobalColor changes, the token numbers again start from one and hence the token numbers used can only grow up to $N$, where $N$ is the number of processes. We refer the reader to [15] for a complete exposition of the algorithm.

\section{Solving GME with bounded registers}

In this section, we generalize the ideas developed by Taubenfeld in [15] and solve the GME problem using bounded registers in linear time and space.

Our algorithm also uses a multi-writer, multi-reader shared bit variable called GlobalColor (see Fig. 4) which can only be black or white. All other shared variables used in the algorithm can only be written by one process even though they can be read by multiple processes. Each process uses a shared variable called Token that has three components viz., session, color and number. We assume that processes can read or write into this Token variable atomically even though it has three components. This is not an unreasonable assumption as this can be implemented without the aid of any higher level synchronization primitives by encoding three integers into a single integer using simple techniques (which we are not elaborating further here). Finally each process also has a boolean shared variable called Choosing. Unlike the GlobalColor 

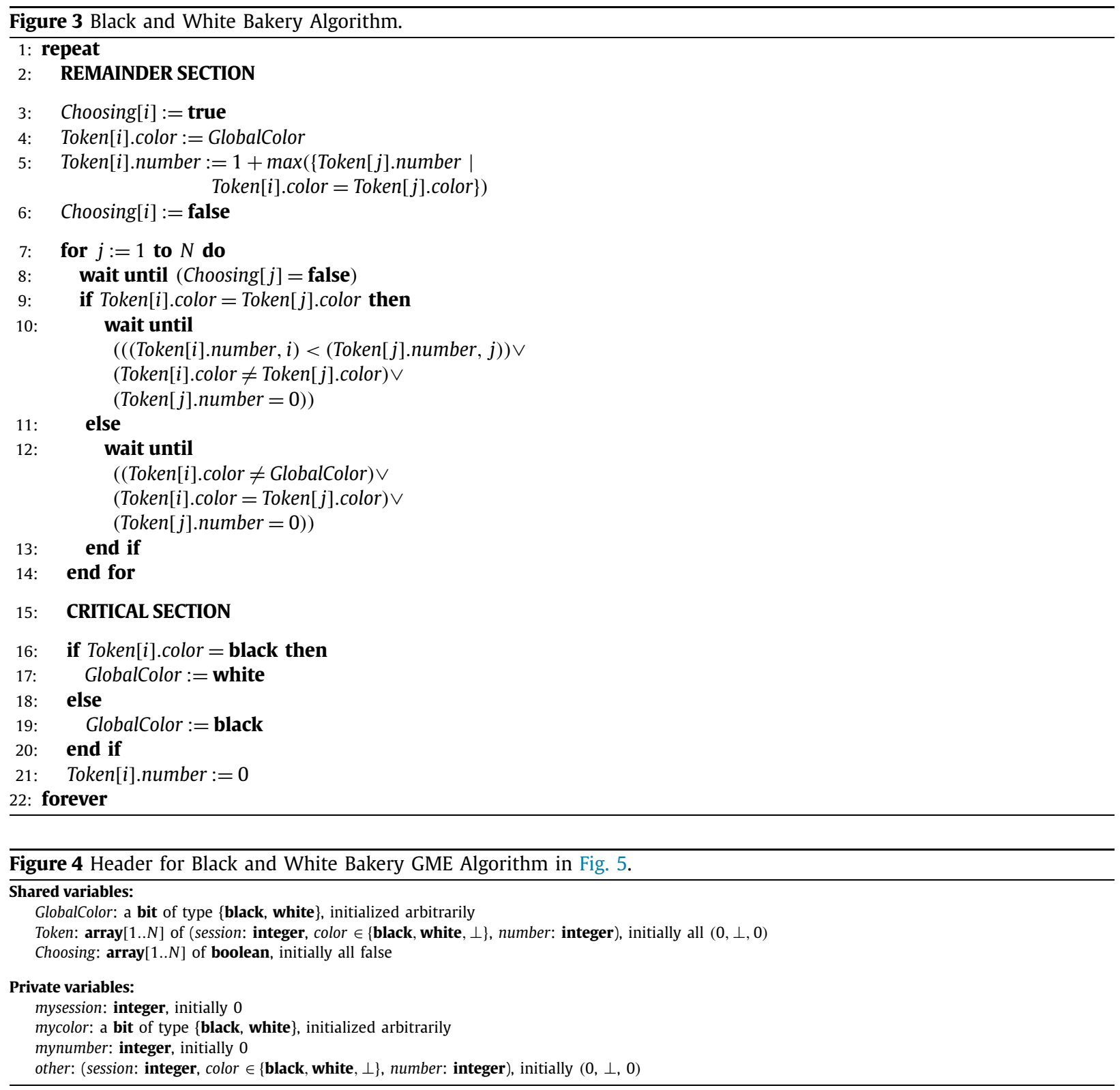

variable, the token color of a process can be black or white or a special value denoted by $\perp$ which indicates that the process has not yet set its token color.

The algorithm is depicted in Fig. 5 and we will refer to it as the Black and White Bakery GME (BWBGME) Algorithm. The doorway of the algorithm is made up of lines 3-15 and the waiting room section consists of lines 16-23. When a process leaves the remainder section, it picks a session number and then updates its Token variable to reflect it. It then sets its Choosing variable to be true to notify other processes that it has initiated the task of picking a token. The token color is set to be the same as the current value of the shared variable GlobalColor. The token number is set to be one more than the maximum of token numbers of conflicting processes with the same color and is set to be 1 in case there are no conflicting processes with the same color. The process then updates its Token variable to reflect the chosen color and number (line 14). It then sets its Choosing variable to be false to notify other processes that it is done with the task of picking a colored token (line 15).

In the waiting room, for each other process $P_{j}$, process $P_{i}$ checks to see if it is an active conflicting process. If it is not, then there is no problem and $P_{i}$ does not wait on $P_{j}$. If it is, then process $P_{i}$ waits until $P_{j}$ has completed selecting its token color and number, if it has initiated the task (line 17). Process $P_{i}$ then checks whether it has priority over $P_{j}$ (lines 18-22). If so, it does not wait on process $P_{j}$ and otherwise it waits on $P_{j}$. The priority order between conflicting processes is the same as that in the Black and White Bakery Algorithm. 

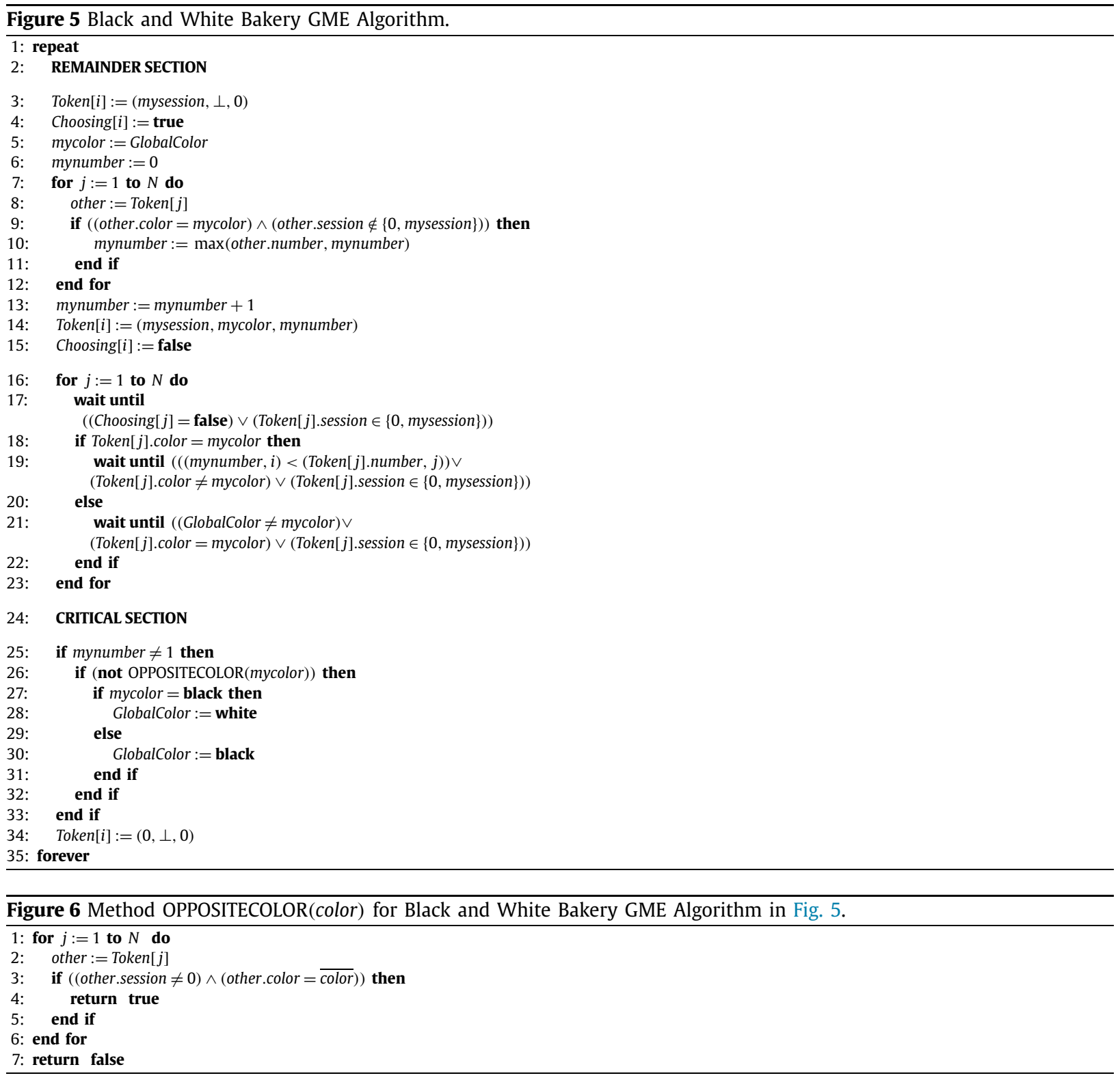

At the time of exiting, process $P_{i}$ checks whether its token number is 1 (line 25). If so, it just resets its Token variable to the initial value and exits. If not, it checks whether there is an active process (not necessarily a conflicting process) with the opposite token color (see Fig. 6). The opposite token color, denoted by color, is defined to be black if color $=$ white, and vice versa. If so, it just resets its Token variable to the initial value and exits. If not, then it updates the GlobalColor to the opposite of its token color and then resets its Token variable to the initial value. In particular, note that token color is reset to $\perp$. This is important to ensure that other processes do not erroneously use a process's old token color while determining the priority.

The generalization of the Black and White Bakery Algorithm to solve the GME problem is quite tricky. While the formal proof of correctness can be found in Section 7, we provide some main insights into our generalization here. In the original Black and White Bakery Algorithm, when a process is attempting to enter the CS, it selects its token color as the current global color and its token number to be one more than the maximum of token numbers of processes with the same color. When it exits the CS, it simply updates the GlobalColor to be the opposite of its own token color. A naive generalization of Black and White Bakery Algorithm would simply add an additional check to see whether the other process is a conflicting process in all busy-wait loops. This naive generalization, nevertheless, cannot ensure the correctness in the case of group mutual exclusion. In the GME, as processes with the same session and different token colors can be in the CS at the same time, if a process leaving the CS simply updates the GlobalColor as before, it may erroneously allow conflicting processes to stay in the CS simultaneously. 
Consider the following scenario. Initially, the GlobalColor is white. Processes $P_{i}$ and $P_{j}$ request the session $S$ and get the token color of white. Then, $P_{i}$ and $P_{j}$ enter the CS concurrently because there is no conflict. When $P_{i}$ is exiting, it sets the GlobalColor to black. Next, a process $P_{k}$ requests the same session $S$ and gets its token color of black. It is easy to see that $P_{k}$ enters the CS by the concurrent entry property. After that, a process $P_{l}$ starts to request a conflicting session $S^{\prime}$ and gets the token color of black. In the waiting room, $P_{l}$ waits for $P_{j}$ since they have different token color and the GlobalColor is black. However, if we let $P_{k}$ exit the CS and then simply set the GlobalColor to white, $P_{l}$ will stop waiting for $P_{j}$ as it sees the GlobalColor is different from its token color. Hence, two conflicting processes $P_{j}$ and $P_{l}$ will be in the CS simultaneously, thus violating the mutual exclusion property.

To get a correct generalization, we observed a key invariant of the original Black and White Bakery Algorithm.

Invariant 1. After a process $P_{i}$ gets its token color from the GlobalColor, the GlobalColor cannot be flipped twice, before the process $P_{i}$ finishes the critical section and gets out of the exit section.

In the original Black and White Bakery Algorithm, suppose a process $P_{a}$ gets a token color of black and after some time the GlobalColor gets changed to white by some process $P_{b}$. Now, in order for some other process $P_{c}$ to change it again to black, $P_{c}$ must be a white process. However, $P_{c}$ can change it only while it exits. In order to exit, $P_{c}$ must first enter the CS. As GlobalColor is currently white and $P_{a}$ has a color of black, $P_{a}$ will have the higher priority over $P_{c}$ to enter the CS. So, the GlobalColor cannot be changed again until $P_{a}$ gets out completely.

The fundamental idea in generalizing the Black and White Bakery Algorithm is to ensure that this invariant is maintained. It is not difficult to see that doing so solves the mutual exclusion violation illustrated in the previous scenario. Although processes $P_{j}$ and $P_{k}$ with different token color stay in the CS at the same time, the GlobalColor will not be updated when $P_{k}$ executes the exit section (as otherwise the GlobalColor is flipped twice since $P_{j}$ got its token color and before it exits) and therefore, $P_{l}$ will still wait for $P_{j}$ until it exits.

In order to maintain this invariant in the generalization, when a process finishes the CS, we let it check whether there is another active process with the opposite token color. If there exists such a process, then the exiting process does not update the GlobalColor. Otherwise, the process updates the GlobalColor to be the opposite color of its own token color. Also, while a process is checking this condition, it may unintentionally access the old token color of another process even though that process has already finished the previous invocation. To prevent this from happening, we let processes reset their token color to empty $(\perp)$ at the end of the exit section.

However, the new GlobalColor updating mechanism is not enough to keep this invariant. A process in the doorway may read the (opposite) GlobalColor and then stop before updating its token color. Hence, another process in the exit section would have no clue as to which color this process will get (it will see a color of $\perp$ for this process). It could erroneously think that there are no active processes with the opposite token color and flip the GlobalColor (while in fact there is an active process with the opposite color). We can devise an intricate sequence of execution to show that the key invariant does not hold if there is such a process that stops just before writing down its token color in the doorway.

In order to handle this, we use a different scheme (from that in the original Black-White algorithm) for token number picking (lines 7-12) and add another condition to check before updating the GlobalColor (line 25). When a process is picking a token number, it determines the maximum of token numbers of conflicting processes with the same token color and then increments it by 1 . If there are no such processes, it selects its token number to be 1 . At the time of exiting, a process does not even attempt to update the GlobalColor if its token number is 1 . On the other hand, if its token number is 2 or more, then it attempts to update the GlobalColor (it actually does if there is no active process with the opposite token color).

These changes are necessary to solve the issue of overlooking a process with the opposite token color having a " $\perp$ " value. Suppose that a hanging process $P_{h}$ has read a value of black for GlobalColor and has not yet written into its token color variable. Suppose that some other process changes the GobalColor from black to white after some time. Later, if a process $P_{u}$ starts to execute the algorithm and attempts to change the GlobalColor again, then $P_{u}$ must be a white-colored process with a token number greater or equal to 2 . In order for process $P_{u}$ to get a token number of 2 or more, there must exist an active conflicting white-colored process $P_{v}$ with a smaller token number. Clearly, one of $P_{u}$ and $P_{v}$ must be in conflict with the hanging process $P_{h}$ because either $P_{u}$ or $P_{v}$ has a different session with $P_{h}$. Therefore, at least one of $P_{u}$ or $P_{v}$ would have waited (in line 17) for $P_{h}$ to finish the token selection before it enters the CS because $P_{h}$ is hanging at the doorway when $P_{u}$ and $P_{v}$ enter the waiting room. Whichever is the case, as $P_{u}$ can enter the CS only after $P_{v}$ has left the $\mathrm{CS}$, we can conclude that the "hanging process" $P_{h}$ has really written down the read GlobalColor to its token variable by the time $P_{u}$ is checking $P_{h}$ 's token color in the exit section. This shows that the scenario that we mentioned before cannot possibly occur anymore. A formal proof that our generalization maintains this key invariant is available in the next section (see Lemma 9).

\section{Proof of correctness of the Black and White Bakery GME Algorithm}

In this section, we present a complete and formal proof of the correctness and complexity analysis of Black and White Bakery GME Algorithm. In particular, we show that our algorithm satisfies the properties P1 through P5, uses only bounded registers and has linear time and space complexity. 
We use the notation $P_{i} @ x$ to denote that process $P_{i}$ is executing line $x$ of the algorithm. The notation $P_{i} @ x \rightarrow P_{j} @ y$ means process $P_{i}$ executes line $x$ before process $P_{j}$ executes line $y$. We use $\overline{\text { color }}$ to denote the opposite color (i.e, $\overline{c o l o r}=$ black if color $=$ white, and vice versa). $\overline{c o l o r}$ is not defined if color $=\perp$. It is easy to see that GlobalColor always has a value of either black or white. Consider the execution of the algorithm on the global time line. We use $I_{i}$ to denote the time interval that the GlobalColor remains as some color after it has been flipped $(i-1)$ times from the beginning. Once the GlobalColor is changed to the opposite color at the end of $I_{i}$, the GlobalColor remains as that color during the interval $I_{i+1}$ We use $t_{i}$ to represent the time point at which the GlobalColor is flipped to the opposite color at the end of $I_{i}$. Note that, $t_{i}$ will be the starting point of the time interval $I_{i+1}$. Process $P_{t_{i}}$ denotes the process that flips the GlobalColor at $t_{i}$.

Here is an example to show a possible execution of the algorithm.

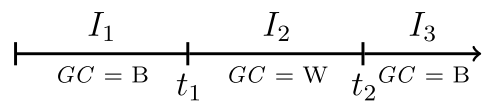

At the beginning, the GlobalColor is initialized to black, and it remains black in the time interval $I_{1}$. At the time point $t_{1}$, the GlobalColor is set to white by a process $P_{t_{1}}$. Then, the GlobalColor remains as white in the time interval $I_{2}$. Note that we are not claiming that the GlobalColor will not be updated during $I_{2}$. However, the GlobalColor will not be updated to black during $I_{2}$ by the very definition of the interval $I_{2}$. The GlobalColor remains as white in the time interval $I_{2}$ until a process $P_{t_{2}}$ sets it to black at time point $t_{2}$.

Lemma 7. If a process $P_{i}$ gets a Token.number of 2 or more, then when $P_{i}$ is calculating its Token.number in the doorway (lines 7-13), there must exist a conflicting process $P_{j}$ with the same Token.color as $P_{i}$ and a smaller Token.number. Moreover, $P_{i}$ cannot enter the CS before $P_{j}$ finishes the $C S$.

Proof. According to line 9, when a process is calculating its Token.number, it will ignore any process with the same session or different Token.color. Assume there doesn't exist such a conflicting process $P_{j}$ with the same Token.color as $P_{i}$ and smaller Token.number. It is easy to see that $P_{i}$ will get a Token.number of 1 , which is a contradiction with $P_{i}$ getting a Token.number of 2 or more. Therefore, such $P_{j}$ must exist when $P_{i}$ is calculating its Token.number.

Moreover, as $P_{j}$ has the same Token.color as $P_{i}$ and a smaller Token.number, $P_{i}$ will wait for $P_{j}$ at line 19 until $P_{j}$ exited the CS and reset its Token. Thus, our claim is true.

Lemma 8. If a process $P_{t_{i}}$ flips the GlobalColor at the time point $t_{i}$, then there must exist a conflicting process $P_{\tilde{t}_{i}}$ with the same Token.color as $P_{t_{i}}$ and a smaller Token.number when $P_{t_{i}}$ was calculating its Token.number (lines 7-13). Moreover, both $P_{t_{i}}$ and $P_{\tilde{t}_{i}}$ execute line 5 in the time interval $I_{i}$.

Proof. Since process $P_{t_{i}}$ flips the GlobalColor at time point $t_{i}$, according to line $25, P_{t_{i}}$ must have the Token.number of 2 or more. By Lemma 7, there must exist a conflicting process $P_{\tilde{t}_{i}}$ with the same Token.color and a smaller Token.number when $P_{t_{i}}$ was calculating its Token.number in the doorway. Next, we show that both $P_{t_{i}}$ and $P_{\tilde{t}_{i}}$ execute line 5 in time interval $I_{i}$

We prove our claim using mathematical induction. Without loss of generality, assume the GlobalColor is initialized to $c$.

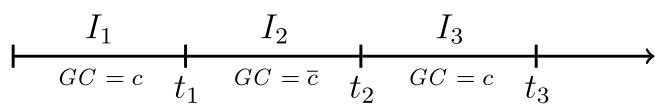

$\mathbf{K}=1$ : Process $P_{t_{1}}$ flips the GlobalColor from $c$ to $\bar{c}$ at $t_{1}$. Obviously, $P_{t_{1}}$ and $P_{\tilde{t_{1}}}$ must have the Token.color of $c$. Since $I_{1}$ is the first and the only time interval that the GlobalColor is $c$ before time point $t_{1}$ and so, $P_{t_{1}}$ and $P_{\tilde{t}_{1}}$ must execute line 5 in $I_{1}$.

$\mathbf{K}=2$ : Process $P_{t_{2}}$ flips the GlobalColor from $\bar{c}$ to $c$ at $t_{2} \cdot P_{t_{2}}$ and $P_{\tilde{t}_{2}}$ must have the Token.color of $\bar{c}$. Since $I_{2}$ is the only time interval that the GlobalColor is $\bar{c}$ before $t_{2}, P_{t_{2}}$ and $P_{\tilde{t}_{2}}$ execute line 5 in $I_{2}$.

Assume the claim holds for $K=(i-1)$.

$\mathbf{K}=\boldsymbol{i}$ : Process $P_{t_{i}}$ may flip the GlobalColor either from $c$ to $\bar{c}$ or $\bar{c}$ to $c$ at time point $t_{i}$. We consider only the case where GlobalColor flips from $c$ to $\bar{c}$ at time point $t_{i}$ as the argument is similar in the other case.

If $P_{t_{i}}$ flips the GlobalColor from $c$ to $\bar{c}$ at $t_{i}$, it is easy to see both $P_{t_{i}}$ and $P_{\tilde{t}_{i}}$ have the Token.color of $c$.

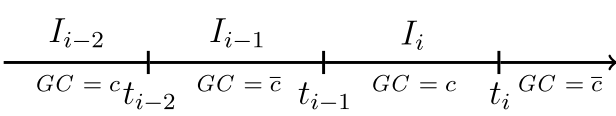


We prove our claim by showing both $P_{t_{i}}$ and $P_{\tilde{t}_{i}}$ execute line 5 in $I_{i}$

1. Assume $P_{t_{i}}$ does not execute line 5 in $I_{i}$, then it must execute line 5 before $t_{i-2}$ since the GlobalColor is $\bar{c}$ during $I_{i-1}$. By induction hypothesis, in time interval $I_{i-1}, P_{t_{i-1}}$ and $P_{t_{i-1}}$ execute line 5 and then write their Token.color of $\bar{c}$. Clearly, $P_{t_{i}}$ must conflict with either $P_{t_{i-1}}$ or $P_{t_{i-1}}$ since $P_{t_{i-1}}$ and $P_{t_{i-1}}$ have different sessions. Therefore, according to line 17, at least one of $P_{t_{i-1}}$ or $P_{t_{i-1}}$ will wait for $P_{t_{i}}$ to finish the doorway to set Choosing $\left[t_{i}\right]$ to false. By Lemma 7 , $P_{t_{i-1}}$ cannot enter the CS before $P_{t_{i-1}}$ finishes the CS. So, when $P_{t_{i-1}}$ enters the CS, $P_{t_{i}}$ has already wrote down its Token.color of $c$ and finished the doorway. After $P_{t_{i-1}}$ finishes the CS and checks the condition at line 26, it will find out that $P_{t_{i}}$ has the opposite Token.color, and so, $P_{t_{i-1}}$ will not update the GlobalColor, which is a contradiction with the assumption that $P_{t_{i-1}}$ flips the GlobalColor at $t_{i-1}$. Therefore, process $P_{t_{i}}$ executes line 5 in time interval $I_{i}$.

2. Since $P_{t_{i}}$ executes line 5 in $I_{i}$, it must read the Token.number of $P_{\tilde{t}_{i}}$ in $I_{i}$, which implies $P_{\tilde{t}_{i}}$ does not reset its Token until $P_{t_{i}}$ reads it in $I_{i}$. Assuming $P_{\tilde{t}_{i}}$ does not execute line 5 in $I_{i}$, it must execute line 5 before $t_{i-2}$. Obviously, $P_{\tilde{t}_{i}}$ conflicts with either $P_{t_{i-1}}$ or $P_{t_{i-1}}$. Therefore, before $P_{t_{i-1}}$ enters the CS, $P_{\tilde{t}_{i}}$ has already written down its Token.color of $c$ and finished its doorway. $P_{\tilde{t}_{i}}$ keeps its Token at least until $P_{t_{i}}$ reads it in $I_{i}$ and this implies $P_{\tilde{t}_{i}}$ keeps its Token when $P_{t_{i-1}}$ is exiting. Hence, in the exit section, $P_{t_{i-1}}$ will find that $P_{\tilde{t}_{i}}$ has the opposite Token.color and so, $P_{t_{i-1}}$ will not flip the GlobalColor, which is a contradiction. So, process $P_{\tilde{t}_{i}}$ executes line 5 in $I_{i}$.

We have shown that if $P_{t_{i}}$ flips the GlobalColor from $c$ to $\bar{c}$ at $t_{i}$, then both $P_{t_{i}}$ and $P_{\tilde{t}_{i}}$ execute line 5 in $I_{i}$. Hence, we have proved our claim for the case $K=i$ and therefore the result follows by mathematical induction.

Lemma 9. After a process $P_{i}$ executes line 5, the GlobalColor cannot be flipped more than once before $P_{i}$ finishes the exit section (line 34).

Proof. Without loss of generality, we assume $P_{i}$ reads the GlobalColor of $c$ at line 5 in the time interval $I_{i}$. Assume that the GlobalColor is flipped twice at time $t_{i}$ and $t_{i+1}$ before $P_{i}$ finishes the exit section. $P_{t_{i+1}}$ represents the process that flips the GlobalColor at $t_{i+1}$.

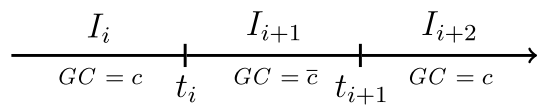

By Lemma 7 and Lemma 8, in $I_{i+1}$, two conflicting processes $P_{t_{i+1}}$ and $P_{t_{i+1}}$ execute line 5 and then finish the CS. So, $P_{i}$ must conflict with either $P_{t_{i+1}}$ or $P_{t_{i+1}}$. Before $P_{t_{i+1}}$ enters the CS, $P_{i}$ must finish the doorway and write down its Token.color of $c$. Thus, after $P_{t_{i+1}}$ has finished the CS, it will find out that $P_{i}$ has the opposite Token.color and fail to update the GlobalColor, which is a contradiction with our assumption that $P_{t_{i+1}}$ flips the GlobalColor at $t_{i+1}$. Hence, we have proved the lemma.

Lemma 10. If process $P_{i}$ and process $P_{j}$ request conflicting sessions and process $P_{i}$ finishes the doorway before process $P_{j}$ executes line 4, then process $P_{j}$ does not enter the CS before process $P_{i}$ finishes the CS.

Proof. For the sake of concreteness, assume process $P_{i}$ gets the Token.color of $c$. Process $P_{j}$ may have a Token.color of either $c$ or $\bar{c}$. We consider both possibilities.

1. If process $P_{j}$ has the Token.color of $c$, it will get a larger Token.number than $P_{i}$ as $P_{j}$ has not begun to calculate its Token.number when $P_{i}$ finishes the doorway. Thus $P_{j}$ will wait for $P_{i}$ at line 19 until $P_{i}$ finishes the CS and resets its Token at line 34 .

2. If process $P_{j}$ gets the Token.color of $\bar{c}$, that means the GlobalColor was flipped to $\bar{c}$ after $P_{i}$ read the GlobalColor of $c$ at line 5. By Lemma 9, the GlobalColor cannot be flipped again to $c$ until $P_{i}$ finishes the exit section. Hence, $P_{j}$ will find out it has the different Token.color from $P_{i}$ and waits for it at line 21. None of the conditions in line 21 can become true until $P_{i}$ finishes the CS and resets its Token[i].

We have shown that in all cases, process $P_{j}$ cannot enter the CS before process $P_{i}$ finishes the CS.

Theorem 2. The BWBGME algorithm satisfies the FCFS property.

Proof. If processes $P_{i}$ and $P_{j}$ request conflicting sessions and $P_{i}$ finishes the doorway before $P_{j}$ starts the doorway, by Lemma $10, P_{j}$ cannot enter the CS before process $P_{i}$ enters the CS.

Theorem 3. The BWBGME algorithm satisfies the Mutual Exclusion property. 
Proof. Suppose the algorithm does not satisfy the mutual exclusion property. At some point of time, there exist two processes $P_{i}$ and $P_{j}$ requesting different sessions that are in the CS simultaneously. By Lemma 10, neither $P_{i}$ nor $P_{j}$ can finish their doorway before the other executes line 4 . Thus, processes $P_{i}$ and $P_{j}$ must have an overlap when they execute lines 4-15. Since $P_{i}$ and $P_{j}$ have different sessions, they wait for each other at line 17 to finish the doorway to set Choosing to false before determining which one of them has the priority.

For the sake of concreteness, we assume that process $P_{i}$ enters the CS with the Token.color of $c$. When $P_{i}$ checks line 18 for $P_{j}$, it may have the Token[j].color of $c$ or $\bar{c}$. We analyze both cases.

1. Process $P_{i}$ finds that $P_{j}$ has the same Token.color of $c$. In order for $P_{i}$ to enter the CS, it must have a smaller Token.number in comparison to $P_{j}$. So, process $P_{j}$ cannot enter the CS before $P_{i}$ finishes the CS and resets its Token[i] as it has the larger Token.number, contradicting the assumption.

2. Process $P_{i}$ finds that process $P_{j}$ has the opposite Token.color of $\bar{c}$. There are two possibilities: (I) after process $P_{j}$ reads the GlobalColor of $\bar{c}$ at line 5, the GlobalColor is flipped to $c$ and then process $P_{i}$ executes line 5 get the Token[i].color of $c$ or (II) after process $P_{i}$ reads the GlobalColor at line 5 of $c$, it is flipped to $\bar{c}$ and then process $P_{j}$ reads it at line 5 and gets the Token[j].color of $\bar{c}$. In order for process $P_{i}$ enter the CS, it must find out the GlobalColor is $\bar{c}$ when it checks line 21 . Therefore, by Lemma 9, only case II can be true. On the other hand, when process $P_{j}$ executes line 21, it will find that none of the conditions are satisfied because the GlobalColor cannot be flipped to $c$ again by Lemma 9 . Therefore, $P_{j}$ will wait for $P_{i}$ until it finishes the exit section, contradicting the assumption that $P_{j}$ and $P_{i}$ stay in the CS at the same time.

We have proved that in all cases we get a contradiction with our assumption, and hence, the algorithm satisfies the Mutual Exclusion property.

Theorem 4. The BWBGME algorithm satisfies the Bounded Exit property.

Proof. Since the exit section does not contain any busy-wait loop, a process that enters the exit section will finish it within a bounded number of its own steps.

Theorem 5. The BWBGME algorithm satisfies the Concurrent Entry property.

Proof. If a process $P_{i}$ requests a session, and no other process requests a different session, for every other process $P_{j}$, it holds that Token[j].session $\in\{0$, mysession $\}$ from the view of $P_{i}$. Since process $P_{i}$ always checks Token $[j] . s e s s i o n$ $\in\{0$, mysession $\}$ in all busy-wait lines (lines 17,19 and 21 ), $P_{i}$ will not wait at any line when there is no conflict and so, it enters the CS within a bounded number of its own steps.

Theorem 6. The BWBGME algorithm satisfies the Deadlock Freedom property.

Proof. Suppose the algorithm does not satisfy the deadlock freedom property. There is an execution of the algorithm in which a nonempty set $S$ of processes enter the entry section but none of them enters the CS, and no process enters the CS infinitely often. As every process in the entry section will finish the doorway eventually and set the Choosing to be false, processes in set $S$ cannot wait on line 17 forever. Let $S_{1}$ be the subset of $S$ that consists of processes having the Token.color of $c$, and $S_{2}$ be the subset of $S$ that consists of processes having the Token.color of $\bar{c}$. Since no process enters the CS infinitely often, the GlobalColor cannot be changed infinitely often. Assume the GlobalColor remains as $c$ after some time.

We observe that processes in $S_{2}$ cannot wait on line 21 forever, because any process in $S_{2}$ will find that their Token.color is different from the GlobalColor and stop waiting. So, processes in $S_{2}$ can only wait on line 19. Also, we observe that processes in $S_{2}$ cannot wait for any process in $S_{1}$ on line 19, because processes in $S_{2}$ will find that they have different Token.color with processes in $S_{1}$ at line 19 , which will make processes in $S_{2}$ immediately terminate waiting. Thus, a process in $S_{2}$ must wait for another process in $S_{2}$ at line 19 . In the set $S_{2}$, there must be a process that has the smallest Token.number and that process will pass all other processes in $S_{2}$ at line 19 and enter the CS. This situation contradicts with our assumption that processes in $S$ cannot enter the CS. So $S_{2}=\emptyset$.

On the other hand, a process in $S_{1}$ cannot wait for any other processes in $S_{1}$ at line 21 because it will find they have the same Token.color and stop waiting. Therefore, every process in $S_{1}$ must wait for another process in $S_{1}$ at line 19 . In the set $S_{1}$, there must exist a process that has the smallest Token.number among all processes in $S_{1}$ and that process will pass all other processes in $S_{1}$ and enter the CS, which is a contradiction. So $S_{1}=\emptyset$.

Hence, we have $S=S_{1} \cup S_{2}=\emptyset$, which contradicts with our assumption and the theorem is proved.

Theorem 7. The BWBGME algorithm satisfies the Starvation Freedom property.

Proof. Lamport has proved that [13] if an algorithm satisfies the deadlock freedom property and the FCFS property, then it necessarily satisfies the starvation freedom property. Hence, using Theorem 2 and Theorem 6, we infer that the algorithm has the starvation freedom property. 
Theorem 8. The shared variables used in the BWBGME algorithm are bounded.

Proof. Obviously, every shared variable used in the algorithm is bounded except possibly Token.number and Token.session. However, if at all the Token.session variable is unbounded, it is due to the nature of the application and not due to the design of the algorithm. In other words, if the underlying application uses only a bounded number of sessions, so will our algorithm. Hence, we need to concern ourselves with only Token.number variable. Here we show that the value of Token.number cannot be larger than $N+1$.

If not, there must exist an execution sequence

$$
\begin{aligned}
P_{i_{1}} @ 5 \rightarrow P_{i_{1}} @ 13 \rightarrow & P_{i_{2}} @ 13 \rightarrow P_{i_{3}} @ 13 \rightarrow \ldots \\
& \rightarrow P_{i_{j}} @ 13 \rightarrow \ldots \rightarrow P_{i_{N+2}} @ 13
\end{aligned}
$$

such that all processes in the sequence have the same Token.color of $c$, and for all $j$ except $1, P_{i_{j}}$ gets its Token $\left.i_{j}\right]$.number at line 13 by incrementing the previous largest number Token $\left[i_{j-1}\right]$.number. $P_{i_{1}}$ gets its Token.number of 1 by virtue of it not finding any conflicting process with the same Token.color when it executes lines 7-12. It is easy to see that for any $j, P_{i_{j}}$ and $P_{i_{j+1}}$ have different session numbers as $P_{i_{j+1}}$ increments $P_{i_{j}}$ 's Token.number. Here we prove the theorem by showing that such a sequence doesn't exist.

We first show that every process in the sequence (1) executes line 5 in the same time interval $I$.

If not, there exist two processes in the sequence, say $P_{i_{k}}$ and $P_{i_{k+1}}$ that execute line 5 in different intervals. As they have the same Token.color, after one of them executes line 5, the GlobalColor is flipped twice before the other process executes line 5. By Lemma 9, the previous process already resets its Token before the GlobalColor is flipped twice, and therefore, $P_{i_{k+1}}$ will fail to read $P_{i_{k}}$ 's Token.number and increment the number, which contradicts our assumption that every process in the sequence (1) increments the previous largest Token.number. So, every process in the sequence (1) executes line 5 in the same interval $I$.

Since we only have $N$ processes, by the pigeonhole principle, at least two processes, say $P_{i_{x}}$ and $P_{i_{y}}$ (not necessarily distinct processes, but with $x<y)$, from the above sequence $\left(P_{i_{1}}, P_{i_{2}}, \ldots, P_{i_{N+2}}\right)$ must exit their CS before $P_{i_{N+2}}$ executes line number 3. So, we have

$$
P_{i_{x}} @ 34 \rightarrow P_{i_{y}} @ 34 \rightarrow P_{i_{N+2}} @ 3
$$

It is easy to see that every two adjacent processes in sequence (1) have different sessions, hence, for any $j, P_{i_{j+1}}$ cannot enter the CS before $P_{i_{j}}$ completely exits and resets its Token $\left[i_{j}\right]$. Consequently, we can conclude that $P_{i_{1}}$ and $P_{i_{2}}$ must have exited by the time $P_{i_{y}}$ exits (as $P_{i_{1}}$ and $P_{i_{2}}$ are the first two processes in the sequence $1, P_{i_{x}}$ and $P_{i_{y}}$ are some processes in the sequence (1) and the processes in the sequence (1) exit one after the other). Thus, before $P_{i_{N+2}}$ begins to execute the doorway, $P_{i_{1}}$ and $P_{i_{2}}$ must already have finished their exit section, which is represented by the following sequence.

$$
P_{i_{1}} @ 34 \rightarrow P_{i_{2}} @ 34 \rightarrow P_{i_{N+2}} @ 3
$$

By our previous claim, $P_{i_{1}}, P_{i_{2}} \ldots P_{i_{N+2}}$ execute line 5 in the same interval and so they all get the same color, say $c$. In order for $P_{i_{1}}$ to enter the CS, all processes conflicting with $P_{i_{1}}$ in the system, having Token.color of $\bar{c}$ must have already finished the exit section and reset their Token. In order for $P_{i_{2}}$ to enter the CS, all processes conflicting with $P_{i_{2}}$ in the system, having Token.color of $\bar{c}$ must have already finished the exit section and reset their Token. Every process that has the Token.color of $\bar{c}$ must be in conflict with either $P_{i_{1}}$ or $P_{i_{2}}$, as these two processes have different session numbers. Hence, after $P_{i_{2}}$ finishes the CS and checks the condition at line 26, it will find that there is no process with the opposite Token.color of $\bar{c}$ in the system. So, $P_{i_{2}}$ will flip the GlobalColor to $\bar{c}$, which immediately starts a new time interval $I^{\prime}$. This contradicts our previous claim that every process in the sequence (1) executes line 5 in the same interval $I$ (as $P_{i_{N+2}}$ in particular will execute line 5 in a different interval).

So, we have shown that such a sequence of processes cannot exist and so proved that the value of the shared variable Token.number is bounded by $N+1$.

Theorem 9. The BWBGME Algorithm has $O(N)$ shared space complexity and $O(N)$ RMR Complexity under the CC model.

Proof. It is trivial to observe that algorithm is of $O(N)$ shared space complexity. Therefore, we focus on proving the RMR complexity here. From line 17 through line 22, there are three busy-wait loops, one each at lines 17, 19 and 21. Since line 19 and line 21 are located in different branches, it is not difficult to see that a process that goes through lines 17-22 executes only two busy-wait loops viz., line 17 and line 19 or line 17 and line 21.

In line 17 , when a process $P_{i}$ is busy waiting for a process $P_{j}$, if Choosing $\left.j\right]$ changes to false, then process $P_{i}$ will immediately terminate the wait. It is possible that process $P_{j}$ executes a new invocation and resets the Choosing $[j]$ to true again with another conflicting session. However, in that case, since process $P_{i}$ doorway proceeds $P_{j}$, according to the FCFS property, $P_{j}$ cannot reenter the CS before $P_{i}$. So, this can occur at most once. Thus, line 17 can only involve at most five RMR (three for Choosing $[j]$ and two for Token $[j]$ ).

Suppose process $P_{i}$ in the entry section executes line 19 and waits for a process $P_{j}$. If process $P_{j}$ changes its Token[j], it will be either resetting its Token, or getting a new Token in a new invocation. If process $P_{j}$ resets its Token[j], $P_{i}$ will 
immediately terminate the wait because it will detect Token $[j]$.session is 0 . However, it is possible that $P_{j}$ wants to reenter the CS with a new Token before $P_{i}$ detects that $P_{j}$ is in the remainder section. In view of the FCFS property, process $P_{j}$ cannot reenter the CS before process $P_{i}$ enters the CS. So, sooner or later process $P_{i}$ will be able to compare its Token[i] with that of process $P_{j}$. Therefore, the maximum number of RMR at line 12 is two (for Token[j]).

Suppose process $P_{i}$ in the entry section executes line 21 and waits for another process $P_{j}$. Without loss of generality, assume $P_{i}$ gets the Token.color of $c$. If the GlobalColor is set to $\bar{c}$, then process $P_{i}$ will find out GlobalColor $\neq$ mycolor and stop the wait. Note that GlobalColor cannot be changed twice to $c$ again when process $P_{i}$ is waiting at line 21 in view of Lemma 9. Hence, process $P_{i}$ will be able to detect the fact that the GlobalColor has changed sooner or later.

It is important to realize that if the variable GlobalColor is overwritten, its cached copies will immediately be invalidated, even if the actual value of the GlobalColor does not really change. This could potentially cause multiple RMR when a process $P_{i}$ is waiting in line 21. Suppose that the GlobalColor is set again to $c$ by another process $P_{k}$ with the Token[k].color of $\vec{c}$ executing the exit section. Now, this situation will cause $P_{i}$ to migrate the GlobalColor to cache again, but $P_{i}$ will continue to be blocked as the value of GlobalColor has not been really changed. In that case, by Lemma $9, P_{k}$ cannot start a new invocation and set the GlobalColor to $c$ again before $P_{i}$ enters the CS. This is because, if $P_{k}$ gets its Token.color of $\bar{c}$ in the new invocation, the GlobalColor is actually flipped to $\bar{c}$ after $P_{i}$ executes line 5 . When $P_{k}$ finishes the CS in the new invocation, it will find out $P_{i}$ has the opposite Token[i].color of $c$ and fail to set the GlobalColor. So, $P_{i}$ will see that the GlobalColor is updated, but still get blocked at line 21 for at most $N-1$ times in the whole loop lines 16-23. Hence, the amortized RMR complexity of GlobalColor for each process $P_{j}$ is constant.

For other conditions at line 21, if process $P_{j}$ resets the Token[j], $P_{i}$ will be able to pass process $P_{j}$. It is possible that $P_{j}$ may want to reenter the CS before $P_{i}$ detects that $P_{j}$ is in the remainder section. In the new invocation, process $P_{j}$ may execute line 3 and write Token $[j]$ of (mysession, $\perp, 0$ ) and block $P_{i}$ again. However, by the FCFS property, process $P_{j}$ cannot reenter the CS before process $P_{i}$ and so $P_{i}$ will be able to detect the new Token[j] or that the GlobalColor is flipped when process $P_{j}$ finishes the doorway, which would make process $P_{i}$ stop waiting. Hence, the overall RMR made at line 21 is constant.

Since the RMR complexity of lines 17,19 and 21 is constant and lines 17-22 are enclosed within a for loop that can run a maximum of $N$ times, it follows that the waiting room (lines 16-23) is of $O(N)$ RMR complexity.

It is trivial to see that lines 8-11 costs constant RMR and so, lines 7-12 is of $O(N)$ RMR complexity. It is not difficult to see that the function used in the algorithm is of $O(N)$ RMR complexity. It is also seen that other lines involve constant RMR. Hence, the overall RMR complexity of this algorithm is $O(N)$ in the CC model.

We summarize the results by stating the properties of our algorithm in the following theorem.

Theorem 10. The BWBGME Algorithm presented in Fig. 5 solves the GME problem by satisfying all the five properties P1 through P5 in linear space (with bounded registers) and time (RMR under the CC model) using only simple read and write operations.

\section{Conclusions and open problems}

We presented two algorithms for solving the GME Problem. Both our algorithms satisfy all the five properties P1 through P5, use only simple read and write operations, run in linear time and linear space. Our first algorithm made use of unbounded shared registers whereas the second algorithm used only bounded shared registers. There is no algorithm in the literature that achieves the combination of both linear time and space complexity while satisfying all five properties in the model in which we are working. As a side contribution, we clarified a flaw in the literature.

Even though the token numbers in our BWBGME algorithm are bounded, the bound is $N+1$ which is not a constant. We leave the development of a linear time and linear space GME algorithm satisfying all five properties, which uses only simple read and write instructions and whose shared registers are bounded by a constant, as an open problem.

Although the five properties mentioned in the problem statement given in the section 1 are the most important properties, there is no end to the wish list of desirable properties. We now discuss some other esoteric properties considered in the literature.

The FCFS property captures fairness across processes requesting different sessions, but not across processes requesting same sessions. This fairness property is captured by the first-in-first-enabled (FIFE) property, described below. The FIFE property was first introduced by Fischer et al. [5] in the context of $l$-exclusion problem.

P7 FIFE: If process $P_{i}$ doorway precedes process $P_{j}$ and the two processes request the same sessions, and $P_{j}$ enters the CS before process $P_{i}$, then $P_{i}$ enters the CS within a bounded number of its own steps.

Another interesting property is the so-called Strong Concurrent Entry (SCE) property. It was first stated in [8] and is a strengthening of the basic concurrent entry property. This property is desirable as late processes (even if they are conflicting) cannot prevent a process from entering the CS within a bounded number of its own steps.

P8 SCE: If a process $P_{i}$ has completed its doorway, and $P_{i}$ doorway precedes every active process that requests a different session from that of $P_{i}$, then $P_{i}$ enters the CS within a bounded number of its own steps. 
Our algorithms do not satisfy the FIFE property or the strong concurrent entry property. It would be nice to develop algorithms that satisfy the FIFE property and SCE property in addition to having all the desirable properties of our BWBGME algorithm stated in Theorem 10. We leave that as an open problem. In [8], a couple of algorithms that additionally satisfy the FIFE property and SCE property are developed by using the notion of abortable mutual exclusion. In [3] an algorithm satisfying FIFE property in addition to other properties is developed. However, none of them runs in bounded linear space. Moreover, these algorithms use the "co-begin co-end construct" with the additional assumption that if one co-routine terminates naturally, all the other coroutines are aborted, thus deviating from the simple model of a process that we are working with in this paper.

\section{Acknowledgements}

The authors would like to thank an anonymous referee who found a subtle error in an earlier version of our Black and White Bakery GME algorithm. The authors would also like to thank Alex Aravind for pointing out a typo in the Black and White Bakery GME Algorithm that was published in the conference version [7].

\section{References}

[1] V. Bhatt, C-C. Huang, Group mutual exclusion in $O(\log n)$ RMR, in: Proceedings of the 29th Annual ACM Symposium on Principles of Distributed Computing, PODC'10, 2010, pp. 45-54.

[2] James E. Burns, Complexity of Communication Among Asynchronous Parallel Processes, Ph.D. thesis, Georgia Institute of Technology, 1981.

[3] R. Danek, V. Hadzilacos, Local-spin group mutual exclusion algorithms, in: Proceedings of the 18th International Symposium on Distributed Computing, DISC'04, in: Lecture Notes in Comput. Sci., vol. 3274, Springer-Verlag, 2004, pp. 71-85.

[4] E. Dijksra, Solution of a problem in concurrent programming control, Commun. ACM 8 (9) (1965) 569.

[5] M. Fischer, N. Lynch, J. Burns, A. Borodin, Resource allocation with immunity to limited process failure, in: Proceedings of the 20th IEEE Annual Symposium on Foundations of Computer Science, FOCS'79, 1979, pp. 234-254.

[6] V. Hadzilacos, A note on group mutual exclusion, in: Proceedings of the 20th Annual ACM Symposium on Principles of Distributed Computing, PODC'01, 2001, pp. 100-106.

[7] Yuan He, K. Gopalakrishnan, E. Gafni, Group mutual exclusion in linear time and space, in: Proceedings of the 17th International Conference on Distributed Computing and Networking, ICDCN'16, ACM, New York, NY, USA, ISBN 978-1-4503-4032-8, 2016, Article No. 22.

[8] P. Jayanti, S. Petrovic, K. Tan, Fair group mutual exclusion, in: Proceedings of the 22nd Annual ACM Symposium on Principles of Distributed Computing, PODC'03, 2003, pp. 278-284.

[9] P. Jayanti, K. Tan, G. Friedland, A. Katz, Bounding Lamport's Bakery Algorithm, in: Proceedings of the 28th Conference on Current Trends in Theory and Practice of Informatics, SOFSEM'01, in: Lecture Notes in Comput. Sci., vol. 2234, Springer-Verlag, 2001, pp. 261-270.

[10] Y. Joung, Asynchronous group mutual exclusion, Distrib. Comput. 13 (4) (2000) 189-206.

[11] Patrick Keane, Mark Moir, A simple local-spin group mutual exclusion algorithm, in: Proceedings of the 18th Annual ACM Symposium on Principles of Distributed Computing, PODC'99, 1999, pp. 23-32.

[12] L. Lamport, A new solution of Dijkstra's concurrent programming problem, Commun. ACM 17 (8) (1974) $453-455$

[13] L. Lamport, The mutual exclusion problem: parts I and II, J. ACM 33 (2) (1986) 313-348.

[14] M. Takamura, Y. Igarashi, Group mutual exclusion algorithms based on ticket orders, in: Proceedings of the 9th Annual International Computing and Combinatorics Conference, COCOON'03, in: Lecture Notes in Comput. Sci., vol. 2697, Springer-Verlag, 2003, pp. $232-241$.

[15] G. Taubenfeld, The Black-White Bakery Algorithm, in: Proceedings of the 18th International Symposium on Distributed Computing, DISC'04, in: Lecture Notes in Comput. Sci., vol. 3274, Springer-Verlag, 2004, pp. 56-70.

[16] J.H. Yang, J. Anderson, A fast, scalable mutual exclusion algorithm, Distrib. Comput. 9 (1) (1995) 51-60. 